\title{
EVOLUÇÃO E NOVAS PERSPECTIVAS PARA A CONSTRUÇÃO E PRODUÇÃO DE INDICADORES DE CIÊNCIA, TECNOLOGIA E INOVAÇÃO ${ }^{1}$
}

\author{
EVOLUTION AND NEW PERSPECTIVES FOR THE DESIGN AND \\ PRODUCTION OF SCIENCE, TECHNOLOGY AND INNOVATION \\ INDICATORS
}

Milena Yumi Ramos - myramos@trieste.fapesp.br

Mestre em Administração

Analista de Indicadores de Ciência, Tecnologia e Inovação da FAPESP

\begin{abstract}
Resumo
Este artigo discute a evolução da produção de estatísticas e indicadores de ciência, tecnologia e inovação, ressaltando as mais importantes contribuições, limitações e perspectivas de aperfeiçoamentos futuros dos indicadores de $\mathrm{P} \& \mathrm{D}$, inovação e novas experiências (indicadores compostos, de posicionamento e de rede). Observa-se -- sob o pano de fundo da dinâmica e estrutura de governança dos sistemas de pesquisa e inovação e dos contextos teórico e tecnológico vigentes -- a evolução de indicadores de insumos e resultados agregados ao nível de país para indicadores customizados, capazes de refletir as especificidades, necessidades e objetivos políticos de cada ator ou grupo de atores atuantes em cada sistema.
\end{abstract}

Palavras-chave: Pesquisa e Desenvolvimento. Ciência e Tecnologia. Inovação. Redes de pesquisa e inovação. Indicadores

\footnotetext{
SIGLAS

ESCWA - United Nations Economic and Social Commission for Western Asia.

EUROSTAT - European Statistical Institute

JRC - Joint Research Center

NISTEP - National Institute of Science and Technology Policy (Japão).

OCDE - Organização para a Cooperação e o Desenvolvimento Econômico; em inglês: Organization for Economic Co-operation and Development.

PRIME - European Network of Excellence on Policies for Research and Innovation in a Move towards The European Research Area

UNDP - United Nations Development Program

UNESCO - United Nations Educational, Scientific and Cultural Organization
}

\footnotetext{
1 Uma primeira versão deste artigo, intitulada: "Evolução, tendências recentes e desafios à construção de indicadores de Ciência, Tecnologia e Inovação", foi apresentada no VII Congreso Iberoamericano de Indicadores de Ciencia y Tecnología - 'nuevos indicadores para nuevas demandas de información', organizado pela Rede Iberoamericana de Indicadores de Ciência e Tecnologia (RICYT) e pela FAPESP, e realizado entre 23 e 25 de maio de 2007 em São Paulo, Brasil.
} 


\section{INTRODUÇ̃̃O}

Nas últimas décadas, os sistemas de pesquisa e inovação têm experimentado mudanças significativas, que, no conjunto, refletem uma nova dinâmica e estrutura de governança. Instituições clássicas, tais como ministérios e secretarias de estado, universidades e institutos de pesquisa e empresas, passaram a diversificar suas estratégias, modos de operação, portfolio de atividades e posição relativa nos sistemas. Em adição, novas instituições começaram a emergir, aumentando sua heterogeneidade e complexidade. Entre elas, pode-se citar: consórcios de pesquisa; joint ventures de vários tipos; plataformas de transferência de tecnologia; start ups; serviços intensivos em conhecimento; empreendimentos de capital de risco; um variado conjunto de instituições de atuação local (agências de fomento, clusters, parques tecnológicos, ONGs com atividades voltadas à Ciência e Tecnologia, etc.); e amplas redes regionais e internacionais de colaboração especializadas.

Frente a essa nova dinâmica, o entendimento da função desempenhada pela inovação nos processos de crescimento econômico, desenvolvimento e redução das disparidades entre países e regiões desiguais pelos diversos setores da sociedade evoluiu para um patamar central e determinante. Na prática, a inovação passou a integrar políticas de Estado, programas de pesquisa das agências governamentais e estratégias de crescimento empresariais, com conseqüências importantes sobre os estilos de gestão pública e privada, que, impulsionados pelo extraordinário avanço das tecnologias de informação e comunicação, passaram a basear-se, muito mais do que no passado, em dados e informações relevantes, acurados e confiáveis, continuamente atualizados e de fácil acesso.

Nesse contexto, os trabalhos no campo das estatísticas e indicadores de ciência, tecnologia e inovação (CT\&I) ganharam um novo impulso. Já são visíveis os resultados do expressivo desenvolvimento que esse campo tem experimentado nas últimas décadas, alavancados pelo surgimento de novos usuários e novas demandas de informação, bem como pelos importantes avanços metodológicos e difusão de tecnologias inéditas. Novas famílias de indicadores, tais como os indicadores compostos, de posicionamento e de rede, têm sido propostas, buscando incorporar uma nova lógica para medir e explicar os fenômenos em curso.

Importante frisar que não se trata de substituir os indicadores clássicos, frutos de anos de esforços de desenvolvimento e produção sistemática por parte dos institutos nacionais de estatísticas e organismos multilaterais de coordenação. A questão-chave não é a falta de qualidade e relevância desses indicadores, mas a inadequação dos conceitos subjacentes para o atendimento das novas demandas de informação e novos usos requeridos. Trata-se, sim, de manter os esforços de aperfeiçoamento dos indicadores existentes, bem como de incitar debates e promover o desenvolvimento de novos indicadores.

Nesse sentido, o primeiro passo é, sem dúvida, revisitar o conhecimento acumulado sobre a produção, uso, limitações e perspectivas de aperfeiçoamento de estatísticas e indicadores clássicos de CT\&I (indicadores de pesquisa e desenvolvimento, de recursos 
humanos em ciência e tecnologia, de produção científica, de patentes, de comércio de produtos com conteúdo tecnológico e, mais recentemente, de inovação - seções 2.1 a 2.3).

O passo seguinte é conhecer as novas propostas para a mensuração das atividades científicas, tecnológicas e inovativas mais destacadas atualmente (indicadores compostos, de posicionamento e de rede - seção 2.4).

Por fim, é importante apresentar e comentar brevemente os principais desafios à evolução da produção de estatísticas e indicadores de CT\&I (seção 3), em busca de um melhor e mais pleno entendimento do processo de inovação.

\section{EVOLUÇÃO DA PRODUÇÃO DE INDICADORES DE CIÊNCIA, TECNOLOGIA E INOVAÇÃO}

O ano de 2006 marcou, segundo Lepori e Godin (2006), um século da coleta e produção sistemática de estatísticas e indicadores de CT\&I no mundo. Nesse período, o desenvolvimento dessas atividades pode ser dividido em três grandes fases, com focos específicos e diferenciados: de 1906 a 1930, a comunidade científica e a produção de conhecimento constituíam o núcleo dos esforços de mensuração; no período póssegunda guerra mundial até fins da década de 80 , passaram a ocupar essa posição as atividades de pesquisa e desenvolvimento institucionalizadas e sistemáticas; e a partir de meados da década de 90, as atividades de inovação tornaram-se o fenômeno observado central. Obviamente tal divisão é meramente didática. Em realidade, as fases se sobrepõem umas às outras e as famílias e tipos de estatísticas e indicadores prevalecentes nas anteriores permanecem em uso e são, inclusive, aperfeiçoados nas seguintes.

Sete aspectos são essenciais no processo de produção de estatísticas e indicadores de CT\&I (BARRÉ, 2006): a lógica subjacente; as necessidades de informação dos usuários; as especificações e concepção; a metodologia e técnicas aplicadas aos conceitos para a construção; a coleta de dados e produção; o armazenamento; a interpretação e o uso. Nas subseções que se seguem, procura-se abordar brevemente esses aspectos, na medida do possível, detalhando um pouco mais cada uma daquelas fases.

\subsection{As primeiras estatísticas de C\&T: tamanho e desempenho da comunidade científica}

Durante a primeira fase de compilação sistemática de estatísticas de C\&T, que marcou o período de 1906 a 1930, os esforços, pioneiramente realizados nos Estados Unidos e depois no Canadá e Grã-Bretanha, voltaram-se para a mensuração do tamanho da comunidade científica (contagem de cientistas) e sua produtividade (contagem de publicações científicas). Em outras palavras, a ciência era medida com base no conceito de conhecimento, através de seus produtos: artigos científicos e, mais tarde, patentes (MACHLUP, 1962; DENISON, 1962; PRICE, 1963; SCHMOOKLER, 1966 apud GODIN, 2004). As análises, então, recaíam sobre a demografia, a geografia e o desempenho dos cientistas. 
Nessa época, essas análises eram conduzidas pelos próprios cientistas, segundo uma visão de que "o limite das atividades de pesquisa e desenvolvimento é fixado pela disponibilidade de pessoal treinado, e não pelo montante de dinheiro disponível",2, e cujo objetivo declarado era melhor gerenciar laboratórios industriais e mobilizar cientistas para a guerra. A principal meta, porém, viria a tornar-se, em pouco tempo, o apoio à formulação e monitoramento da política científica em nível nacional.

\subsection{Indicadores de Pesquisa e Desenvolvimento (P\&D)}

A partir dos anos de 1920, e mais intensamente no pós-guerra (1945-1990) a pesquisa passou a ser entendida como importante driver da prosperidade econômica. Considerava-se pesquisa, então, toda a atividade que inexoravelmente envolvesse o investimento de recursos escassos, sobretudo financeiros e recursos humanos qualificados, para a produção de conhecimento, com o objetivo geral de aumentar a produtividade futura, em sentido mais amplo (GODIN, 2004). Assim, não apenas as universidades, mas todos os outros setores econômicos envolvidos nessas atividades indústria, governo, e organizações sem fins lucrativos - constituíam as unidades de análise.

Em razão desse ponto de inflexão na importância da pesquisa para o desempenho econômico, os governos nacionais transformaram-se no principal setor a demandar sistematicamente informações para o planejamento de orçamentos para a ciência e para a mensuração dos recursos públicos aplicados em pesquisa (LEPORI e GODIN, 2006; GODIN, 2006). Acima de tudo, buscavam dimensionar a eficiência ou produtividade do sistema de ciência, definida como resultados obtidos com as atividades de pesquisa. Por detrás desse modo de mensuração, portanto, estava a adoção do modelo input-output, segundo a perspectiva das Contas Nacionais.

Nesse momento, a produção de estatísticas de $\mathrm{P} \& \mathrm{D}$, bem como o desenvolvimento de novas estatísticas e indicadores e fontes de dados associadas, tornaram-se institucionalizadas, e passaram a ser conduzidos por departamentos e institutos nacionais de estatística, que tomaram para si a responsabilidade de atender às novas necessidades de informação que se apresentavam.

Foram realizadas, então, as primeiras experiências com surveys em atividades de pesquisa e desenvolvimento, conduzidas de maneira ad hoc, através da compilação do maior número de dados disponíveis ou da coleta de informações por meio de questionários com poucas instruções aos respondentes. Padrões para surveys surgiram apenas a partir dos anos de 1950 nos Estados Unidos (pela National Science Foundation) e, logo em seguida, em outros países via OCDE (Manual Frascati - OECD, 1963) e, em menor grau, via UNESCO (1984).

$2 \quad$ Traduzido do inglês: "the ceiling on research and development activities is fixed by the availability of trained personnel, rather than by the amounts of money available..." (US President Scientific Research Board, 1947 in LEPORI e GODIN, 2006). 
Operacionalmente, de início, utilizou-se o conceito de pesquisa $(\mathrm{P})$ nesses surveys. Posteriormente, observando-se o papel central que o desenvolvimento tecnológico desempenhava nas empresas e na agenda política, as atividades de desenvolvimento experimental foram adicionadas às de pesquisa (P\&D). Oficialmente, de acordo com a revisão mais recente do Manual Frascati (OECD, 2002), compõem as atividades de $\mathrm{P} \& \mathrm{D}$ aquelas voltadas à criação de conhecimento novo, normalmente associadas à pesquisa básica, e aquelas voltadas ao desenvolvimento de novas aplicações baseadas no conhecimento acumulado, geralmente associado à pesquisa aplicada e ao desenvolvimento experimental.

Destaque-se que esse conceito adotado para definir as estatísticas de $\mathrm{P} \& \mathrm{D}$, então, referia-se às atividades de pesquisa e desenvolvimento institucionalizadas e realizadas sistematicamente (GODIN, 2004). Uma vez que apenas grandes laboratórios industriais e instituições públicas de pesquisa correspondiam a esta definição, uma grande parte da pesquisa não era coberta pelos surveys. Era o caso da pesquisa realizada em pequenas empresas $^{3}$, por exemplo. Somavam-se a tal viés dois outros: o fato de os surveys concentrarem-se na pesquisa realizada no setor industrial (em detrimento do de serviços, do público, etc.) e nas inovações tecnológicas (em detrimento das organizacionais, de mercado, etc.).

Nesses surveys de $\mathrm{P} \& \mathrm{D}$, o principal aspecto investigado eram os gastos ou dispêndios brutos e relativos alocados às atividades de pesquisa e desenvolvimento. Em termos agregados, sua razão com relação ao produto interno bruto tornou-se o principal indicador nas análises em nível nacional.

A composição desse indicador para fins de cômputo dos gastos totais de um país em $\mathrm{P} \& \mathrm{D}$, sobretudo para efeito de comparações internacionais em bases padronizadas, foi sendo aprimorada, até atingir a atual composição ${ }^{4}$ (ESCWA, 2003). Tais indicadores

3 Pequenas empresas raramente possuem departamentos de P\&D separados e dificilmente separam orçamentos especialmente para P\&D. Mesmo estando engajadas em tais atividades, muitas vezes não as consideram P\&D quando respondem aos surveys. Por outro lado, grande parte das atividades de P\&D, normalmente mais D do que P, nessas empresas, é conduzida de maneira ad hoc e informal, freqüentemente incorporada às suas atividades produtivas, e não em bases regulares (SMITH, 1998).

(a) gastos empresariais em P\&D (Business Enterprise $R \& D$ Expenditure - BERD), que representam o somatório das contribuições às atividades de $\mathrm{P} \& \mathrm{D}$ conduzidas por empresas, organizações e institutos privados cuja função primordial é a produção e comercialização de produtos e serviços, exceto ensino superior, bem como as instituições sem fins lucrativos que as atendem. As contribuições à P\&D por empresas públicas também são incluídas nesta categoria; (b) gastos do governo em P\&D (Government $R \& D$ Expenditure - GERD), que incorporam a soma das execuções em P\&D de agências, departamentos e outras entidades que oferecem produtos e serviços públicos, exceto ensino superior, bem como daquelas que fiscalizam as ações do governo. Este indicador também inclui gastos das instituições sem fins lucrativos supervisionadas pelo governo; (c) gastos de Instituições de Ensino Superior em P\&D (Higher Education R\&D Expenditure - HERD), que representam o somatório dos gastos em P\&D realizados por instituições de ensino superior públicas e privadas de qualquer natureza jurídica. Também estão incluídos nesta categoria os gastos de centros de pesquisa, estações experimentais e clínicas vinculadas a instituições de ensino superior; (d) gastos de empresas privadas sem fins lucrativos em P\&D (Private Non-profit R\&D Expenditure - PNPERD), que inclui a soma dos gastos em P\&D realizados por instituições sem fins lucrativos que servem ao setor público, bem como aqueles realizados com recursos provenientes de doadores; e (e) gastos a partir de contribuições extranacionais - em recursos financeiros 
têm sido amplamente utilizados em exercícios de benchmarking entre países, e também para monitorar o progresso, ou falta dele, nas atividades de P\&D ao longo do tempo, além de comparar o apoio à $\mathrm{P} \& \mathrm{D}$ nos diversos setores da atividade econômica dentro de um mesmo país.

Outro indicador de insumo bastante disseminado refere-se aos recursos humanos em C\&T. Diferentemente da prática adotada nos primórdios da compilação de estatísticas de C\&T, não só os "cientistas", mas também outros indivíduos empregados em atividades de $\mathrm{P} \& \mathrm{D}$ ou correlatas, tais como diretores, gerentes e outros, passaram a ser contabilizados.

Também com relação a essa família de indicadores, à semelhança dos de gasto, a atuação de organismos internacionais, destacadamente da OCDE, tem sido marcante. Em 1995, a Organização elaborou recomendações, até certo ponto complementares àquelas contidas no Manual Frascati, para a mensuração dos recursos humanos dedicados à ciência e tecnologia (RHCT), compiladas no Manual de Canberra (OECD, 1995). Tal manual adota uma concepção mais ampla para os RHCT, incluindo todas as pessoas com elevada qualificação - das quais uma parcela efetivamente se dedica a atividades de P\&D -, cujo papel de elo entre as práticas cotidianas e as atividades inovativas é decisivo. Segundo essa abordagem, compõem os RHCT todas as pessoas que completaram o ensino pós-secundário ou que trabalhem em ocupações associadas à ciência e tecnologia, ainda que não tenham completado esse nível de ensino.

Entendidos como os carreadores da capacidade tecnológica e inovativa, presente e futura, os RHCT têm sido considerados um tema central na produção de indicadores no setor. São utilizados, em geral, para dimensionar o número de trabalhadores altamente qualificados em um determinado país ou região, seu nível de habilidades e treinamento, e a taxa segundo a qual esse estoque estratégico cresce ou decresce ao longo do tempo.

Tanto os indicadores de gastos em P\&D quanto de RHCT medem o volume de recursos ou insumos para as atividades de pesquisa e desenvolvimento. À medida que a atuação governamental nesse setor foi se ampliando, os usuários de tais indicadores começaram a questionar o valor de medidas, como essas, relacionadas exclusivamente aos insumos. O provimento de informações quantitativas sobre potenciais benefícios em termos de resultados de $\mathrm{P} \& \mathrm{D}$, tendo em vista uma satisfatória orientação à formulação de políticas públicas e estratégias empresariais, além das estatísticas e indicadores de insumos, passou, então, a fazer parte das agendas de pesquisa e trabalho de especialistas no setor.

Conforme esses debates caminhavam para um consenso, iam sendo introduzidas nos esforços de produção de estatísticas e indicadores de P\&D fontes de dados de produtos desse conjunto de atividades. Uma das principais fontes, ainda hoje, são os bancos de dados de patentes 5 .

ou físicos - em P\&D (Extra-national Contributions), que totalizam as contribuições para P\&D de organismos e indivíduos estabelecidos fora do país em questão.

À semelhança do esforço empreendido pela OCDE com relação à harmonização de metodologias de compilação e interpretação de estatísticas e indicadores de P\&D e de RHCT, a 
Outra é o conjunto de bases de dados de publicações científicas ${ }^{6}$. A partir dessas fontes, tendo como balizadores, por exemplo, o tamanho da população em geral e do pessoal em $P \& D$, puderam ser produzidos indicadores para avaliar a intensidade de pesquisa de países e instituições (ESCWA, 2003), que, em última análise, davam uma medida do volume de conhecimento acumulado e do grau de sucesso e de especialização de um país em relação a outros.

Com a intensificação dos fluxos de comércio e tecnologia entre os países, que acompanhou a ascensão dos movimentos de globalização da economia e da internacionalização das atividades de $\mathrm{P} \& \mathrm{D}$, uma nova categoria de indicadores de resultado tornou-se popular: indicadores de transferência de tecnologia, captados através do balanço de pagamentos tecnológico (BPT). Esses indicadores cobrem a compra e venda de tecnologias desincorporadas - tais como direitos de propriedade, licenças, assistência técnica - e também pagamentos não relativos à tecnologia, nomeadamente serviços de gestão (ESCWA, 2003). Dessa forma, refletiam, a depender das metodologias e procedimentos de coleta e tratamento de dados utilizados, o fluxo de investimentos em propriedade intelectual, o ritmo da difusão tecnológica e o nível de competitividade entre setores selecionados e países em análises comparadas.

Novamente, com relação a essa categoria de indicadores, a OCDE posicionou-se ativamente, coletando e publicando informações e dados em BPT desde 1982. Posteriormente, em 1990, lançou o chamado TBP Manual (OECD, 1990), no qual propunha métodos para a compilação e interpretação dessa família de dados.

$\mathrm{Na}$ fase mais recente de produção de estatísticas de $\mathrm{P} \& \mathrm{D}$, especialmente nos últimos 15 anos, houve um esforço de coleta e divulgação dessas estatísticas em bases regulares, procurando seguir recomendações metodológicas internacionais, sobretudo na forma de publicações de caráter panorâmico, apresentando múltiplos indicadores desenvolvidos até então, acompanhados de interpretação e análise contextualizadas (scoreboards, outlooks). A novidade, segundo Godin (2004), foi que os esforços de construção de indicadores concentraram-se nos produtos e alguns resultados das atividades de P\&D: titulações conferidas (graduação e pós-graduação), artigos publicados, patentes depositadas e concedidas, exportações e importações de produtos com conteúdo tecnológico.

Embora tenha experimentado um extraordinário avanço principalmente na segunda metade do século XX, a produção de indicadores de $\mathrm{P} \& \mathrm{D}$ não encontrou uma solução definitiva para algumas limitações importantes. A mais destacada delas refere-se ao fato

Organização propôs, em 1994, um conjunto de orientações sobre como obter e analisar estatísticas de patentes e, a partir delas, criar indicadores de patentes. Tais orientações foram compiladas no documento conhecido como Patent Manual (OECD, 1994).

Essas fontes de dados, e os indicadores bibliométricos construídos a partir delas, diferentemente de todas as outras famílias de indicadores de CT\&I, mais comuns em publicações panorâmicas acerca da situação atual e tendências no setor, não foram tomados como objeto de trabalho por parte de organismos internacionais envolvidos na produção de estatísticas, mas sim foram abarcadas por grandes grupos privados, com destaque para o grupo ISI Thomson. 
de que dados de insumos para $\mathrm{P} \& \mathrm{D}$ agregados ao nível da economia como um todo suprimem informações importantes sobre a composição industrial das atividades de P\&D, o mix de pesquisa básica, aplicada e de outros tipos, e o mix de pesquisas financiadas com recursos públicos e privados, e assim por diante (EUROSTAT, 1997).

Não obstante tal limitação, os indicadores de P\&D e o modelo linear subjacente, alavancado pela ciência, representam, até os dias de hoje, fontes importantes de informação para a construção de panoramas e para a orientação de políticas públicas no setor de C\&T. Seu sucesso deve-se, em parte, à existência de conceitos e metodologias consolidados, dados históricos e internacionalmente comparáveis. Não por isso, obviamente, deixam de ser alvo do "problema das notas de rodapé" (FREEMAN E SOETE, 2007), que dizer de ressalvas à interpretação considerando diferenças entre os países em definições, classificações, metodologias de cálculo adotadas, características estruturais, tais como tamanho da economia, estrutura industrial, e também de estudos para futuros aperfeiçoamentos.

\subsection{Indicadores de inovação}

Até poucos anos atrás, na década de 80 , a inovação era simplesmente concebida como uma progressão linear de uma fase inicial de concepção de uma idéia, normalmente associada à pesquisa básica, até sua tradução numa efetiva aplicação. Branscomb apud ESCWA (2003), chamou essa concepção linear de inovação de "modelo de funil" (pipeline model). De acordo com esse modelo, para promover a inovação duas estratégias são centrais: minimizar gargalos e manter uma rica oferta de insumos, nomeadamente recursos financeiros e pessoal qualificado para P\&D.

Contudo, esse modelo "entrada = pesquisa e saída = tecnologia", começou a ser questionado, por um lado, com o acirramento da globalização, a transição da economia e da sociedade para um novo paradigma baseado no conhecimento e o extraordinário avanço e difusão das tecnologias de informação e comunicação; e, por outro, com a retomada da teoria Schumpeteriana.

Schumpeter apud Arundel e Hollanders (2006) separava invenção (concepção de uma nova idéia) de inovação (comercialização de um novo produto ou implementação de um novo processo). Esta separação de conceitos encontrou duas barreiras no modelo vigente: primeiro, as invenções devem ser testadas no mercado para que se possa captar efetivamente seu valor de mercado; e segundo, as empresas podem inovar com base em esforço criativo próprio limitado ou mesmo inexistente, como acontece quando adquirem novas tecnologias. Tal avanço conceitual impôs novas necessidades quanto à construção de indicadores de resultados, devendo estes refletir os processos de difusão e adoção de novos produtos e de ganhos de produtividade em cada setor econômico.

Diante dessas novas necessidades, em 1992, os países da OCDE estabeleceram um acordo para o desenvolvimento de estatísticas próprias para medir a inovação, com o objetivo de prover dados relevantes sobre todo o espectro das atividades inovativas conduzidas por empresas, incluindo P\&D e outras formas segundo as quais elas podem inovar. O primeiro passo nesse sentido foi a publicação do Manual de Oslo (OECD, 
1996), que continha diretrizes para a coleta de dados via surveys de inovação ${ }^{7}$, como Community Innovation Survey (CIS) no âmbito da União Européia, e sua interpretação.

A proposta desses surveys era superar um sério gargalo apresentado pela geração anterior de dados sobre C\&T disponíveis, qual seja quantificar a inovatividade geral da economia, levando em conta as interações entre atores e instituições. Nessa nova geração de dados, os fluxos de conhecimento nos sistemas de pesquisa e inovação, inclusive através da mobilidade do capital humano, tornaram-se uma variável-chave para identificar e caracterizar suas forças e deficiências e apoiar a elaboração de análises mais aprofundadas capazes de gerar recomendações para melhorar o desempenho desses sistemas ${ }^{8}$.

É inegável que esses desenvolvimentos teóricos e metodológicos proporcionaram um avanço considerável do entendimento e mensuração do processo de inovação; no entanto, importantes limitações ainda se impõem à sua consolidação.

Por se tratar da mensuração de um fenômeno complexo, multifacetado e que proporciona impactos intangíveis, difusos e muitas vezes perceptíveis apenas no longo prazo, diversas críticas, presentes já desde os primeiros trabalhos com indicadores de inovação, têm-se apresentado. Algumas das mais recorrentes estão relacionadas (ARUNDEL et alii, 1998; SMITH, 1998; ESCWA, 2003; GODIN, 2006): ao escopo da definição de inovação nos surveys; à mensurabilidade das diversas atividades que compõem o processo de inovação; à correspondência entre as abordagens adotadas para medir insumos e produtos das atividades de inovação 9 ; à real dimensão dos resultados das atividades inovativas, sobretudo com respeito à melhoria do desempenho geral das empresas e do país; ao entendimento prévio das razões que levam as empresas a inovarem e fatores que estimulam e obstaculizam o processo de inovação, e de sua evolução no tempo, como pré-requisito à realização de comparações internacionais e intersetoriais.

\footnotetext{
7 Esses surveys buscam investigar aspectos do processo de inovação: fontes de informação relevantes para a inovação, insumos (recursos financeiros, P\&D, desenho industrial, inteligência de mercado e outros), objetivos da inovação, transferência e aquisição de tecnologia, cooperação para a inovação, fatores promotores e resultados das atividades inovativas (número e volume de venda de produtos novos ou aperfeiçoados), obstáculos à inovação, adotantes ou usuários externos, uso de tecnologias-chave, tais como TICs, métodos para proteger a inovação, apoio governamental, etc. As metodologias internacionais para os surveys de inovação usualmente definem-na segundo a perspectiva Schumpeteriana, e focam apenas produtos significativamente aperfeiçoados ou totalmente novos, enquanto que outras abordagens incluem, também, inovações incrementais, de pequena escala.

Essa premissa, entretanto viria a ser contestada mais tarde: evidências empíricas mostraram que: (a) nem sempre o movimento de recursos humanos qualificados de fato significa uma transferência real de conhecimento de uma disciplina ou instituição para outra; e (b) nem sempre esses fluxos de conhecimentos estão correlacionados com a eficiência do processo de inovação (Arundel et alii, 1998).

A esse respeito, a CIS, por exemplo, capta, como resultados das atividades de inovação, a parcela de produtos novos ou aperfeiçoados na carteira global da empresa. Porém, capta, ao mesmo tempo, do lado dos insumos, o total disponível para todo o tipo de inovação resultante; ou seja, as abordagens não correspondem (EC, 2003).
} 
Adicionalmente, a lógica subjacente aos indicadores de inovação mantém, na prática, a linha adotada no período precedente: a unidade de análise - a empresa (em particular a industrial) - é investigada em termos de insumos aplicados (indicadores de input), produtos derivados (indicadores de output) e resultados econômicos alcançados (economic outcome) (GODIN, 2002). Os agregados nacionais correspondentes, nesse caso, refletem os resultados acumulados das empresas localizadas dentro das fronteiras geográficas do país.

E ainda faltam evidências empíricas capazes de reforçar sua adequação frente às novas teorias de inovação e dinâmica recente dos sistemas de C\&T. Freqüentemente as séries temporais de inovação existentes são incompletas e apresentam inconsistências em função de alterações na metodologia de coleta de dados e de cálculo. A solução mais viável encontrada para superar essas dificuldades tem sido o uso de proxies para captar alguma(s) dimensão(ões) de análise, com conhecidas conseqüências para sua capacidade de retratar fielmente o processo de inovação.

Um exemplo bastante ilustrativo dos problemas gerados por essas aproximações é o uso de indicadores de patentes ${ }^{10}$ como proxy dos resultados do processo de inovação. Se, por um lado, os indicadores de patentes apresentam a vantagem de alto nível de desagregação dos dados, possibilitando a investigação ao nível de empresas individuais (EUROSTAT, 1997); por outro, alterações nos níveis de insumos às atividades de P\&D, possivelmente detectadas por indicadores de input, não necessariamente são explicações exclusivas para mudanças nos níveis de patenteamento. Pode haver explicações associadas a questões legais, administrativas, ou à disponibilidade de outros meios de apropriação dos lucros sobre os investimentos em P\&D, ou ainda a mudanças na quantidade de inovações com potencial de mercado, entre outros.

Portanto, a produção sistemática de indicadores de inovação encontra-se em estágio embrionário mundialmente, haja vista o recente desenvolvimento do arcabouço conceitual e metodológico sobre o qual estão embasados e as poucas experiências, ainda em franca evolução, de compilação de dados a partir de surveys. Para a maioria dos países em desenvolvimento esses indicadores constituem, ainda, uma novidade (ESCWA, 2003). Não obstante, ao lado do GERD e GERD/GDP, têm se tornado, com o amparo de análises e pesquisas complementares, as estatísticas mais utilizadas pelos gestores públicos no setor de C\&T.

\subsection{Experiências recentes e perspectivas de construção de novos indicadores}

A partir das idéias fundamentais de Schumpeter (1949), o conceito do processo de inovação vem evoluindo e, atualmente, assume a forma de um modelo freqüentemente referido como interativo, com ampla aceitação entre teóricos e especialistas. As múltiplas interações ocorrem em uma vasta e diversa rede de atores envolvidos na criação, difusão e uso do conhecimento científico e tecnológico, e tal dinâmica

10 Alguns autores (ARUNDEL e HOLLANDERS, 2006) defendem que os indicadores de inovação atualmente em uso, tais como patentes, representam resultados intermediários do processo de inovação. 
proporciona um conjunto de insumos que, eventualmente, transforma-se em mudança inovativa.

Note-se que a concepção interativa do processo de inovação introduziu, explicitamente, o papel determinante dos fluxos de conhecimento ${ }^{11}$ entre instituições e atores (EUROSTAT, 1997) na família de indicadores de insumo, junto aos recursos financeiros e humanos tradicionalmente considerados. Por outro lado, trouxe um obstáculo importante para a construção e interpretação de indicadores de inovação (Arundel et alii, 1998): os processos de criação, difusão e uso de conhecimentos podem acontecer ao mesmo tempo e são interdependentes, gerando um problema de fronteira de difícil equacionamento, tendo em vista os instrumentos e metodologias de coleta, tratamento e análise de dados atualmente disponíveis.

Em uma perspectiva microeconômica, segundo esse modelo, a P\&D não está confinada exclusivamente, e não necessariamente nem mesmo principalmente, na origem do processo, mas é conduzida em todos os estágios do processo de inovação, desde a concepção de uma idéia, análise de mercado, desenvolvimento e design, teste, produção, marketing, distribuição até aperfeiçoamentos subseqüentes do produto. Nesse tipo de conceito, ganham importância atividades informais de $\mathrm{P} \& \mathrm{D}$, de caráter ad hoc, adicionalmente à P\&D formal (SMITH, 1998).

Diante desses desenvolvimentos teóricos, dos significativos avanços e ampliação do acesso às tecnologias digitais, e da entrada de novos atores nos sistemas de pesquisa e inovação, pondo em questão a adequação dos indicadores existentes e impondo uma pressão positiva por indicadores de CT\&I alternativos, novos players $^{12}$ foram atraídos para a esfera de construção e produção desses dados (BARRÉ et alii, 2007).

Como resultado dessa diversificação no campo dos indicadores de CT\&I, em termos de produtores, usuários, demandas e usos, têm emergido, sobretudo a partir de fins da década de 90, um conjunto de novos indicadores de CT\&I, com destaque para os indicadores compostos, de posicionamento e de rede, comentados em mais detalhes a seguir.

\footnotetext{
11 Tais fluxos incluem transferência de tecnologia e fluxos de know-how, seja na forma de spillovers, seja na forma de transferências intencionais. Portanto, não são limitados a trocas de informações entre empresas e instituições (ARUNDEL et alii, 1998). Esses fluxos também estão sujeitos a "externalidades", isto é, à incorporação de conhecimentos absorvidos de outras empresas e instituições, do mesmo campo de atuação ou de outros, bem como à sua recombinação para o desenvolvimento de novas aplicações. Logo, essa teoria assume que o processo de inovação ocorre tanto mais rápido quanto mais atores inovadores se beneficiem do conhecimento acumulado de outros.

12 Dentre eles, incluem-se produtores independentes de indicadores, tais como certos tipos de observatórios de C\&T, entidades profissionais e consultorias privadas; institutos de pesquisa especializados, a exemplo do Center for Science and Technology Studies (CWTS), Leiden University (Holanda); organismos semi-oficiais dedicados com missão de coordenação, como o Institute for Prospective Technological Studies (IPTS), no espaço europeu; além de diversos grupos de pesquisa universitários e centros de pesquisa.
} 
Note-se, porém, que, neste momento, representam exercícios, carecendo, portanto, de toda uma base metodológica estabelecida e de toda uma estrutura de produção sistemática. Pelo contrário, esses indicadores são produzidos de maneira ad hoc, e portanto longe de atender adequadamente aos requisitos de qualidade, regularidade e uso exigidos pelos usuários.

\subsubsection{Indicadores compostos (composite indicators)}

Indicadores compostos são medidas que agregam diversas variáveis associadas a um fenômeno observável multidimensional, entendido sob um determinado quadro teórico, em um único índice. Este representa uma média ponderada de um rol selecionado de componentes $^{13}$, informando a posição relativa, num ranking, de uma determinada entidade (setor, país, região, etc.). Adicionalmente, pode informar, em uma série histórica, a evolução na posição de cada ente ao longo do tempo, provendo informações acerca do seu desempenho geral (EC, 2003).

Índices desse tipo têm sido utilizados com sucesso em exercícios de benchmarking entre países em diversas áreas de atuação de políticas públicas ${ }^{14} \mathrm{com}$ o intuito de identificar tendências gerais e áreas em que o país não alcançou o desempenho desejado e, a partir daí, estabelecer políticas prioritárias e determinar metas de desempenho correspondentes.

No campo da ciência, tecnologia e inovação, a produção de indicadores compostos é recente e tem incorporado quatro principais dimensões (ESCWA, 2003), prevalecendo, em cada caso, aquela mais atinente com a área de atuação política em que a intervenção é mais necessária:

- indicadores relacionados a condições estruturais e de infra-estrutura que determinam a capacidade nacional e das empresas de estabelecer normas e expandir o leque de oportunidades para inovar. Abrangem componentes políticos e físicos, incluindo a comunicação dentro das organizações e entre elas;

- indicadores de capacitação em CT\&I, incluindo a maneira como o conhecimento é gerado e utilizado, a disponibilidade de sistemas de apoio e fomento à pesquisa básica e aplicada e a capacidade de realizar planejamento estratégico de P\&D;

- indicadores relacionados a fatores de desenvolvimento de recursos humanos, incluindo sistemas de educação superior e de treinamento técnico especializado; e

13 A atribuição de pesos, neste caso, segue um entendimento conceitual do fenômeno, compactuado entre os pesquisadores, produtores e usuários do indicador composto, bem como características subjacentes aos dados utilizados, tais como confiabilidade. A soma de componentes, normalmente indicadores simples, que apresentam diferentes dimensões de medida requer, também, procedimentos de normalização (transformações, tratamentos econométricos e estatísticos). Outro ponto crítico freqüentemente levantado na produção desses indicadores refere-se a relações de causa e efeito, muitas vezes indissociáveis, entre as variáveis que os integram.

14 Historicamente, os indicadores compostos têm sido desenvolvidos e utilizados na esfera econômica, tais como o Produto Interno Bruto (PIB), o Índice de Preços ao Consumidor (IPC) e o Índice de Gini. Atualmente, indicadores compostos são utilizados em diversos campos, além da economia, tais como: sociedade; ambiente; inovação, tecnologia, informação; e globalização (ver: $<$ http://farmweb.jrc.cec.eu.int/CI/Indexes.htm>). 
- indicadores relacionados a fatores sociais e culturais que influenciam a forma como a inovação pode ser gerada, acessada e implementada efetivamente pelas empresas.

Tendo em vista essas dimensões, constituem experiências relevantes: o Technology Achievement Index (TAI), das Nações Unidas (UNDP, 2001); o Composite of Innovation Performance, da OCDE (FREUNDENBERG, 2003); o Overall Science and Technology Index, do Japão (NISTEP, 1995); e o Índice Brasil de Inovação (IBI) (Uniemp Inovação, vários números).

Mais recentemente, a Comissão Européia, no âmbito do exercício "Indicadores Estruturais", tem apoiado o desenvolvimento de um conjunto de indicadores compostos para a economia baseada no conhecimento ${ }^{15}$ (SMITH, 2000; JRC, 2002). Diversas unidades da Comissão engajaram-se nessa tarefa, que já produziu dois resultados: um índice de investimento na economia baseada no conhecimento e um índice de desempenho na transição para a economia baseada no conhecimento.

Tais iniciativas apresentam importantes vantagens, sendo as principais (SAISANA e TARANTOLA (2002) apud NARDO et alii (2005)):

- possuir a capacidade de resumir questões complexas ou multidimensionais;

- interpretação mais fácil, comparativamente à identificação de tendências a partir de um conjunto mais numeroso de indicadores simples;

- facilitar a tarefa de posicionar países ou setores em um ranking, considerando exercícios de benchmarking;

- permitir o monitoramento do progresso de países ou setores ao longo do tempo em questões complexas;

- encerrar um mesmo volume de informações em um espaço menor ou encerrar mais informações em mesmo espaço, relativamente ao conjunto de indicadores simples que o compõem;

- colocar a questão do desempenho e do progresso do país ou setor no centro do debate político; e

- facilitar a comunicação com a sociedade em geral e promover a quantificação de importantes fenômenos ligados ao crescimento econômico e ao desenvolvimento social.

Essas vantagens conferem aos indicadores compostos grande valor como instrumento de comunicação e ferramenta de apoio à formulação e monitoramento de políticas públicas e estratégias empresariais, o que os têm tornado bastante populares.

Contudo, alguns autores (FREUNDENBERG, 2003) têm levantado sérias dúvidas sobre sua exatidão e confiabilidade, e colocado em questão as recomendações de políticas derivadas. Os principais questionamentos incluem:

15 Ver projeto Knowledge Economy Indicators (KEI): development of innovative and reliable indicator systems, desenvolvido no âmbito do Sixth Framework Programme on Research and Development da Comissão Européia no período 2004-2007. Mais informações em: $<$ http://kei.publicstatistics.net/>. 
- a incapacidade de um único índice prover toda a informação necessária aos usuários ${ }^{16}$;

- a possibilidade de embasarem recomendações inadequadas para a formulação de políticas públicas, se construídos com pouco rigor ou interpretados indevidamente;

- a possibilidade de incitarem conclusões simplistas com relação a políticas públicas;

- a possibilidade de serem indevidamente utilizados, se o processo de construção não for transparente ${ }^{17}$ e se não houver forte embasamento conceitual ${ }^{18}$ nem tratamento estatístico rigoroso;

- a seleção dos componentes e pesos pode ser alvo de pressões políticas, permitindo manipulações para produzirem os resultados desejados;

- a possibilidade de mascararem sérias falhas em algumas dimensões ${ }^{19}$, aumentando a dificuldade de identificar ações corretivas apropriadas; e

- a possibilidade de levarem ao desenho de políticas não apropriadas se as dimensões de desempenho difíceis de serem medidas forem ignoradas.

Segundo Freundenberg (2003), esses problemas tornam os indicadores compostos sujeitos a usos inadequados, particularmente quando se prestam a listar países ou setores em rankings comparativos ou em séries históricas.

Tendo em vista as importantes vantagens e limitações não desprezíveis que apresentam, diversos autores (DESAI et alii, 2002; NISTEP, 1995) alertam para o fato de que os indicadores compostos devem ser utilizados como ferramentas iniciais dentro de um programa mais amplo de monitoramento e avaliação do desempenho dos países ou setores na criação, difusão e uso de tecnologia e no processo de inovação como um todo. Desse ponto de vista, forneceriam uma visão geral da posição desses entes no cenário internacional da CT\&I, apontando indícios de tendências gerais de desempenho, cujas causas e efeitos, bem como a dinâmica mais ampla em que se inserem, deveriam ser objeto de estudos complementares, com maior profundidade analítica.

16 Basta lembrar que esses índices são construídos a partir de indicadores, com suas limitações intrínsecas, e não de medidas diretas.

17 Por exemplo, quando a metodologia e dados originais não são explicitados aos usuários, estes não podem distinguir entre o desempenho real (avanço ou deterioração em alguma ou todas as áreas) e o aparente, influenciado pelo método e cobertura dos dados, isto é, diferenças no posicionamento de países ou setores em um ranking ao longo do tempo podem resultar de melhoramentos nos dados, diferentes métodos de atribuição de pesos ou outras mudanças metodológicas, e não necessariamente de qualquer mudança no desempenho desses entes. Por isso, evita-se utilizar indicadores compostos para a construção de séries históricas.

${ }_{18}$ Nesse ponto, em particular, deve-se considerar que apesar de alguns componentes poderem desempenhar um papel mais importante que outros no desempenho inovativo de um país ou outra entidade em análise, é difícil estabelecer uma hierarquia. O quadro teórico atualmente disponível para explicar o desempenho inovativo não está suficientemente desenvolvido para que se possa atribuir pesos aos componentes com total segurança.

19 Por exemplo, quando o índice incorpora componentes dependentes de escala, estes devem ser corrigidos a priori, através de técnicas de cálculo sobre os dados originais (método empírico), sob o risco de se incorrer em distorções no cômputo geral (Statistics Canada, 2006). Assim, é importante explicitar as opções de abordagens e técnicas adotadas, as limitações associadas, os componentes escolhidos e sua capacidade de representar cada dimensão considerada na composição do índice. 
Recomendam, também, que o indicador seja desagregado, de modo que os analistas possam investigar o desempenho do ente em cada componente (FREUNDENBERG, 2003), possibilitando a identificação mais precisa de suas forças e fraquezas e o levantamento de hipóteses que expliquem o desempenho geral observado. Outro aspecto que ressaltam é a necessidade de haver maior cuidado na interpretação de rankings construídos com base nesses indicadores, já que muitos deles não informam sobre a liderança no desenvolvimento tecnológico global, mas sim sobre quão bem um país ou setor individual participa dos processos medidos.

\subsubsection{Indicadores de rede (network indicators)}

Com custos de comunicação reduzidos e fronteiras geográficas diluídas, a identificação e mensuração das redes de pesquisa e inovação têm-se tornado um objeto central de discussões entre os produtores de estatísticas e indicadores de CT\&I, sobretudo quando está em jogo o mapeamento de competências científico-tecnológicas e decisões acerca da alocação de recursos financeiros, físicos e humanos.

Nesse contexto, a internacionalização adquire uma dimensão especial. Empresas transnacionais passaram a organizar suas atividades de inovação considerando suas filiais em todo o mundo, tornando pouco útil o relacionamento entre o desempenho inovador em um país e as atividades de inovação conduzidas nesse mesmo país. Com as universidades e institutos de pesquisa engajados em acordos de cooperação tecnológica estabelecidos com parceiros no exterior algo similar, em diferentes graus, tem ocorrido nos últimos anos.

As implicações dessa nova dinâmica para a produção de indicadores de CT\&I são claras, como destaca (BARRÉ, 2006): há uma demanda por novos indicadores (a) referentes a uma grande variedade de entidades, com capacidade de descrever parâmetros relevantes (competências, visibilidade) e relacionamentos (fluxo financeiro, de pessoas, coordenação estratégica), com cobertura subnacional; e (b) referentes à dimensão macrorregional ou supranacional das políticas, o que requer coordenação e até mesmo integração de políticas e instituições, a exemplo do que ocorre no âmbito da União Européia $^{20}$.

Na prática, porém, o arcabouço conceitual, metodológico e instrumental de mensuração não tem acompanhado esses novos desenvolvimentos no mesmo compasso: há uma falta crítica de dados e indicadores sistemáticos que sirvam de insumo para analistas compreenderem a natureza, dinâmica e fatores de sucesso das redes de pesquisa e inovação (ESCWA, 2003). Indicadores de rede em uso atualmente ${ }^{21}$ baseiam-se em proxies, tais como a amplitude das alianças estratégicas, patentes e citações de patentes,

20 Por exemplo, as diversas iniciativas no âmbito dos Framework Programmes on Research and Technological Development. Ver mais detalhes em: <http://ec.europa.eu/research/index.cfm>, e o esforço de consolidação da European Research Area.

$21 \quad$ Para mais detalhes, consultar os trabalhos desenvolvidos no âmbito do projeto Science, Technology, Innovation Network Indicators - STI-NET, sob o programa Improving Human Potential and Socio-Economic Knowledge, da Comissão Européia). Ver: <http://www.stinet.org>. 
publicações científicas e citações (indicadores bibliométricos) e parcerias para pesquisa e desenvolvimento tecnológico.

Num futuro próximo, o que se espera, para essa família de indicadores, é que forneçam subsídios mais realistas para a formulação e monitoramento de políticas públicas em inovação, sendo, então, capazes de informar (EC, 2003):

- o grau com que as redes evoluem de nacionais ou regionais para globais, com implicações importantes para a gestão dos processos de transferência de tecnologia, proteção da propriedade intelectual, concorrência e desenvolvimento regional;

- a competitividade em setores intensivos em conhecimento, analisada com base nas redes empresariais, e não nas empresas individualmente;

- a eficácia dos incentivos e critérios de avaliação estabelecidos pelos governos para a promoção de novas tecnologias com a finalidade de aumentar a eficácia das estruturas de rede; e

- formação de redes público-privadas em setores ou tecnologias específicas.

Nesse sentido, a necessidade de abandonar uma perspectiva estática e definida - uma entidade em um ponto do tempo - em favor de uma perspectiva mais dinâmica diversas entidades heterogêneas interagindo simultaneamente ao longo do tempo permanece, ainda, como um desafio aos produtores de indicadores de rede.

\subsubsection{Indicadores de posicionamento (positioning indicators)}

Historicamente, a maior parte do esforço de pesquisa e do trabalho sobre estatísticas e indicadores de CT\&I tem-se baseado no modelo input-output, segundo a lógica de função de produção presente nos Sistemas de Contas Nacionais. Como conseqüência, o principal nível de análise é o país, encobrindo os agentes individuais e reduzindo os sistemas de inovação a poucos setores agregados ${ }^{22}$, medidos sob diferentes aspectos (LEPORI e GODIN, 2006). O esforço de organismos multilaterais, especialmente da OCDE e do EUROSTAT, para promover e garantir a comparabilidade dos dados entre países e blocos teve um grande impacto sobre essa tendência. A produção sistemática de estatísticas e indicadores de CT\&I, nesse nível, alcançou, assim, a maturidade.

Entretanto, nos últimos vinte anos, tem emergido um conjunto de indicadores que, segundo Barré (2006), alguns têm chamado de indicadores de posicionamento, voltados à caracterização dos elementos dos sistemas de inovação, considerando que estes são compostos por agentes estratégicos, heterogêneos e autônomos, formando um sistema no qual as competências encontram-se distribuídas. Nessa nova lógica, o foco recai não apenas sobre os agentes individuais, mas nas relações que estabelecem entre si (competição, cooperação ou outras), bem como em suas complementaridades, buscando obter um panorama mais acurado dos diferentes tipos e categorias a que pertencem. Em outras palavras, busca-se conhecer o posicionamento e trajetória dos agentes no sistema.

22 Nesta visão macro, os subníveis são constituídos por grupos de instituições categorizados segundo sua propriedade (pública, privada ou sem fins lucrativos); principal objetivo (ensino superior; pesquisa aplicada, desenvolvimento tecnológico, etc.); e pelo produto físico que fornecem à sociedade (definição de setores industriais). 
Uma implicação importante dessa nova família de indicadores é a multiplicação dos níveis de análise possíveis (BARRÉ et alii, 2007). Ao lado dos indicadores nacionais e regionais, indicadores para organizações individuais são demandados por gestores encarregados de formular sua estratégia e monitorar sua realização, bem como por gestores responsáveis por avaliar o desempenho das instituições. Evidentemente, esse tipo de abordagem aumenta muito a complexidade da produção de indicadores de CT\&I (múltiplos tipos de indicadores, problemas metodológicos inerentes à sua construção, fontes de dados requeridas, comparabilidade em diferentes níveis, etc.).

As experiências emergentes de construção de indicadores de posicionamento, desenvolvidas sobretudo na Europa ${ }^{23}$, atestam tal complexidade. Os exercícios realizados até este momento demonstraram que, para um avanço mais significativo da produção e uso desses indicadores, é necessário melhorar sua confiabilidade e relevância, em termos: (a) do alcance de um consenso em relação ao tipo e definição exata dos indicadores de posicionamento, que ainda apresentam conflitos, influenciados pelos objetivos e estratégias próprios dos diferentes atores presentes nos sistemas de pesquisa e inovação; (b) da produção de séries temporais, uma tarefa atualmente impossível, dada a natureza ad hoc das novas bases de dados e do tratamento e reconfiguração das bases de dados já disponíveis; (b) da formação de conhecimento acumulado e memória de métodos de cálculo e de dados, de modo a possibilitar técnica e financeiramente a comparabilidade entre estudos. Atualmente esses requisitos não estão sendo atendidos, sendo necessário recomeçar todo o trabalho de concepção e desenho dos indicadores a cada survey realizado; (c) da incorporação das necessidades da comunidade científica especializada em políticas de CT\&I, fortalecendo a capacidade de produzir conhecimento coletivo em apoio a políticas e estratégias para o setor.

\section{DESAFIOS E PERSPECTIVAS À CONSTRUÇÃO DE INDICADORES DE CIÊNCIA, TECNOLOGIA E INOVAÇÃO}

Tendo em vista o histórico e evolução recente da produção de estatísticas e indicadores de CT\&I, é possível afirmar que o momento atual é de uma profunda revisão dos modelos conceituais utilizados para alicerçar o desenvolvimento de metodologias de mensuração do processo de inovação.

Destaque-se aqui, o papel de vanguarda da OCDE e do instituto de estatística europeu (EUROSTAT) que, em meados da década de 90, como resultado de uma reunião de ministros das pastas de ciência e tecnologia dos países membros, iniciaram um esforço internacional - aberto ao pensamento criativo acerca da mensuração das atividades de inovação ${ }^{24}$ - para a proposição de novas estatísticas e indicadores capazes de incorporar os novos conceitos que passavam a integrar as teorias de inovação e captar os resultados

\footnotetext{
23 Ver, por exemplo, os trabalhos desenvolvidos no âmbito do projeto European Network of Indicator Producers - ENIP, que propõe a criação de uma Plataforma Européia de Indicadores de Ciência, Tecnologia e Inovação (ESTIP, sigla em inglês) baseada nesse tipo de indicadores, disponíveis em: <http://81.80.44.195:15080/enip/sections/estip/>.

24 Essa iniciativa ficou conhecida como "blue sky indicators".
} 
e impactos ${ }^{25}$ dessas atividades, melhor assistindo os gestores públicos e privados (COLECCHIA, 2006; GAULT, 2007).

Balizando esse esforço, estão a apreensão do real significado dos sistemas nacionais de inovação para a construção e análise de estatísticas de CT\&I e a incorporação de perspectivas complementares à econômica na análise do processo de inovação e seus impactos, refletindo no desenvolvimento de novas fontes de dados de natureza multidimensional (considerando aspectos sociais, culturais, ambientais, de saúde).

Os objetivos últimos dessa iniciativa convergem no sentido de: (a) prover assistência direta ao desenvolvimento e implementação de ações políticas concretas, o que pressupõe, em primeiro lugar, um bom entendimento do contexto político, especificamente das opções de políticas disponíveis e das restrições ao desenvolvimento de novas ações políticas; e, em segundo lugar, um bom entendimento do significado de cada estatística e indicador existente ou novo para a formulação de políticas de inovação; (b) apresentar potencial de contribuição para o desenvolvimento das teorias de inovação, num processo contínuo de teste e aperfeiçoamento das teorias existentes; e (c) auxiliar empresas privadas e outras instituições a desenvolverem e ajustarem suas próprias estratégias de inovação, uma característica de auto-reforço, já que estatísticas e indicadores de inovação são tão mais valorizados quanto maior e melhor apoio direto dão àqueles que as utilizam.

A concretização de tais objetivos passa impreterivelmente pela exploração do potencial das novas famílias de indicadores de CT\&I que vêm sendo propostas, impondo aos pesquisadores, produtores e usuários envolvidos nesse campo o enfrentamento de dois desafios centrais: o desafio da capitalização e o desafio da inovação.

Primeiramente, o desafio da capitalização tem a ver com a necessidade de se evitar o risco de recomeçar a cada vez que um novo indicador é desenvolvido de maneira ad hoc, e implica uma transição do desenvolvimento experimental para o acúmulo organizado de competências no que diz respeito a essas iniciativas. Para tanto, é indispensável preconizar a documentação e amplo compartilhamento de metodologias e práticas de produção de novos indicadores de CT\&I, bem como sua reprodutibilidade e comparabilidade.

Mais precisamente, trata-se de fomentar todo um esforço de coordenação de metodologias e conceitos (entre usuários, produtores especializados e academia); de uso de princípios e padrões comuns, permitindo comparações e baixando o custo de produção; de intensificação da interoperabilidade entre os indicadores produzidos de forma descentralizada, e suas fontes; de manutenção de uma regularidade de divulgação adequada; e, finalmente, de compartilhamento do conjunto desses esforços para que os

25 Há, atualmente, um amplo e intenso debate acerca do conceito de impacto das atividades de pesquisa e inovação e de como medi-lo. No entanto, entre os produtores de estatísticas e indicadores de CT\&I tem-se formado um relativo consenso de que se refere a mudanças na economia, na sociedade, em seus valores e comportamentos. Os instrumentos mais apropriados para medir tais impactos seriam surveys específicos, ainda não disponíveis nem padronizados (GODIN, 2006). 
novos indicadores construídos possam ser apropriados e validados por toda a comunidade de produtores e usuários.

No entanto, consolidar um sistema estruturado tal como acima sugerido não é suficiente para garantir um adequado alinhamento entre a produção de indicadores de CT\&I e as demandas de informação e requisitos de uso permanentemente em evolução. Para isso, é imprescindível, também, alimentar a capacidade de desenhar novos indicadores, com base em sólidos fundamentos conceituais e teóricos e no uso "inteligente" das fontes de dados existentes (considerando inclusive suas limitações) isoladamente ou combinadas com fontes não-estatísticas advindas de diversos contextos institucionais. Em outras palavras, é necessário promover a inovação dentro dos sistemas de construção e produção de indicadores de CT\&I.

Para lidar com esses desafios, pesquisadores e especialistas ligados à rede PRIME apontam para a estruturação de um organismo capaz de prover um conjunto de serviços especializados para melhorar a coerência de tal sistema (BARRÉ et alii, 2007). Entre eles, estariam incluídos:

- serviços técnicos, tais como hospedagem de bases de dados produzidas por instituições sem a competência requerida; provisão de treinamento na construção e gerenciamento de tais bases (especialmente para instituições acadêmicas, que não necessariamente são bem equipadas nesse quesito);

- desenvolvimento de regras e padrões para acesso aberto e para a interoperabilidade de dados coletados em campos específicos;

- acumulação de experiência metodológica e provisão de padrões para projetos de indicadores, concernentes ao nível de documentação associados a todos os contratos de indicadores, por exemplo;

- circulação da informação sobre projetos de indicadores existentes e resultados, e provisão de acesso aos documentos e aos dados originais.

Para concluir, embora importantes avanços tenham sido feitos no desenvolvimento de indicadores de CT\&I, muitos desafios de difícil equacionamento se impõem aos produtores desses dados. Ainda será necessário um longo período até que os indicadores já desenvolvidos sejam aperfeiçoados, e que as novas propostas apresentadas nos últimos anos tornem-se robustas e internacionalmente comparáveis. Não obstante, a continuação dos trabalhos nessa direção é absolutamente necessária para um melhor e mais pleno entendimento do processo de inovação.

\section{BIBLIOGRAFIA}

ARUNDEL, Anthony; HOLLANDERS, Hugo. Searching the forest for the trees: "missing" indicators of innovation. Trend Chart methodology report, 2006. Disponível em: http://trendchart.cordis.lu/scoreboards/scoreboard2006/pdf/eis_2006_methodology_rep ort_missing_indicators.pdf $>$, Acesso em: 27/02/2007. 
ARUNDEL, Anthony; SMITH, keith; PATEL, Pari; SIRILLI, Giorgio. The future of innovation measurement in Europe: concepts, problems and practical directions. Idea Report, n.3, 1998. Disponível em:

$<$ http://www.step.no/old/Projectarea/IDEA/Idea3.pdf >, Acesso em: 23 /02/ 2007.

BARRÉ, Remi. The emergency of "positioning indicators": towards a European STI Indicators Platform (ESTIP)?. Paris, France: Carré des Sciences, 6-10 /02/ 2006. Disponível em:

$<$ http://www.primenoe.org/Local/prime/dir/General\%20Presentation/2006\%20Annual\%20Conference/EST IP_position_paper_V1.pdf>, Acesso em: 07 /02/ 2007.

BARRÉ, Remi; FILLIATREAU, Ghislaine; LEPORI, Benedetto. New perspectives for the design and production of S\&T indicators. In: PRIME THIRD ANNUAL CONFERENCE, 29 Jan.- ${ }^{\text {st }}$ Feb. 2007, Pisa, Italy. Paper presented at the... Disponível em:

$<$ http://www.primenoe.org/Local/prime/dir/Annual\%20Conference/2007\%20Annual\%20Conference/Positi on\%20Papers/Position\%20Paper\%20-\%20Indicators.pdf >, Acesso em: 10/09/2007.

COLECCHIA, Alessandra. What indicators for science, technology and innovation policies in the $21^{\text {st }}$ century?. In: BLUE SKY II FORUM - WHAT INDICATORS FOR SCIENCE, TECHNOLOGY AND INNOVATION POLICIES IN THE $21^{\text {ST }}$ CENTURY, 25-27 September 2006, Ottawa, Ontario, Canada. (background paper). Disponível em: <http://www.oecd.org/dataoecd/9/48/37082579.pdf>, Acesso em: 15/02/2007.

DESAI, Meghnad; FUKUDA-PARR, Sakiko; JOHANSSON, Claes; SAGASTI, Fransisco. Measuring the technology achievement of nations and the capacity to participate in the network age. Journal of Human Development, v.3, n.1, p.95-122, 2002.

Disponível

em:

$<$ http://unpan1.un.org/intradoc/groups/public/documents/apcity/unpan014340.pdf $>$, Acesso em: 15/02/2007.

DOMINGUES, Sílvia Angélica; FURTADO, André. Recursos humanos em P\&D. Uniemp Inovação, São Paulo, Ano 2, n.7, p.26-27, 2006. Disponível em: $<$ http://www.labjor.unicamp.br/ibi/arquivos/ibi_ed07.pdf >. Acesso em: 15 /02/ 2007.

EC - EUROPEAN COMMISSION. European report on science \& technology indicators. Luxembourg: Office for Official Publications of the European Communities, 2003. Disponível em: $<\mathrm{ftp}$ :/ftp.cordis.europa.eu/pub/indicators/docs/3rd_report.pdf $>$, Acesso em: 09/02/2007. ESCWA. New indicators for science, technology and innovation in the knowledgebased society. New York, USA: United Nations Publications, 2003. Disponível em: $<\mathrm{http}$ ://www.escwa.org.lb/information/publications/edit/upload/sdpd-03-5.pdf $>$, Acesso em: $12 / 02 / 2007$.

EUROSTAT. Statistics on research and development. In: $3^{\mathrm{TH}}$ CEIES SEMINAR, Aarhus, Denmark, 3-4 December 1997. Disponível em: $<$ http://forum.europa.eu.int/Public/irc/dsis/ceies/library?l=/seminars/statistics_developm ent/procd_en_doc/_EN_1.0_\&a $=\mathrm{d}>$, Acesso em: 01/03/2007.

FRANCO, Eliane; CAR VALHO, Flávia; CARVALHO, Sílvia. Como construir grupos setoriais. Uniemp Inovação, São Paulo, Ano 2, n.5, p.26-27, 2006. Disponível em: $<$ http://www.labjor.unicamp.br/ibi/arquivos/ibi_ed05.pdf $>$. Acesso em: 15/02/2007. 
FREEMAN, Christopher; SOETE, Luc. Developing science, technology and innovation indicators: what can we learn from the past. UNU-MERIT Working Paper Series, n. 2007-001, 2007. Disponível em: $<$ http://www.merit.unu.edu/publications/wppdf/2007/wp2007-001.pdf >, Acesso em: 05/03/2007.

FREUDENBERG, Michael. Composite indicators of country performance: a critical assessment. STI Working Paper. Industry Issues, 2003. Disponível em: $<$ http://www.olis.oecd.org/olis/2003doc.nsf/43bb6130e5e86e5fc12569fa005d004c/8bb0 f462911c2cc6c1256ddc00436279/\$FILE/JT00153477.PDF>, Acesso em: 12/02/2007.

FURTADO, André; QUADROS, Ruy; QUEIROZ, Sérgio. Projeto tem início com empresas do setor indistrial. Uniemp Inovação, São Paulo, Ano 2, n.4, p.26-27, 2006. Disponível em: <http://www.labjor.unicamp.br/ibi/arquivos/ibi_ed04.pdf>. Acesso em: $14 / 02 / 2007$

FURTADO, André; QUEIROZ, Sérgio. Etapas para a criação de um índice. Uniemp Inovação, São Paulo, Ano 2, n.1, p.26-27, 2006. Disponível em: $<$ http://www.labjor.unicamp.br/ibi/arquivos/ibi_ed01.pdf > . Acesso em: 14/02/ 2007

GAULT, Fred. How far and fast can we go?. In: $32^{\mathrm{ND}}$ CEIES SEMINAR INNOVATION INDICATORS: MORE THAN TECHNOLOGY, February 5-6, 2007, Aarhus, Denmark. Disponível em: $<$ http://forum.europa.eu.int/Public/irc/dsis/ceies/library?1=/ceies_seminars_31/indicators technology/documents/en/61_f_gaultpdf/_EN_1.0_\&a=d>, Acesso em: 13/02/2007.

GODIN, Benoit. Measuring output: when economics drives science and technology measurements. Project on the History and Sociology of S\&T Statistics Working Paper, n.14, 2002. Disponível em: <http://www.inrsucs.uquebec.ca/inc/CV/godinpub.htm>, Acesso em: 7/05/2007.

GODIN, Benoît. Renewing S\&T indicators: from R\&D to knowledge. In: $1^{\text {ST }}$ INTERNATIONAL CONFERENCE ON INNOVATION "INNOVATION MANAGEMENT IN A GLOBAL ENVIRONMENT", September 22 - 24, 2004, Moscow, The Russian Federation: ISTC - International Science and Technology Center. Disponível em: < http://www.csiic.ca/PDF/Godin_Moscow.pdf $>$, Acesso em: 27/02/2007.

GODIN, Benoît. Statistics and science, technology and innovation policy: how to get relevant indicators. In: BLUE SKY II - WHAT INDICATORS FOR SCIENCE, TECHNOLOGY AND INNOVATION POLICIES IN THE $21^{\text {ST }}$ CENTURY, 25-27 September 2006, Ottawa, Canada. Disponível em: $<$ http://www.oecd.org/dataoecd/48/15/37483949.pdf>, Acesso em: 07/02/2007.

JRC. State-of-the-art report on current methodologies and practices for composite indicator development. Luxembourg: EC, 2002. Disponível em: $<$ http://farmweb.jrc.cec.eu.int/ci/Document/state-of-the-art_EUR20408.pdf $>$, Acesso em: $12 / 02 / 2007$.

LEPORI, Benedetto; GODIN, Benoît. In: INDICATORS ON SCIENCE, TECHNOLOGY AND INNOVATION: HISTORY AND NEW PERSPECTIVES, 1517 November 2006, Lugano, Switzerland. (background notes). Disponível em: $<$ http://www.ticinoricerca.ch/conference/background.html $>$, Acesso em: 07/02/2007.

NARDO, Michela; SAISANA, Michaela; SALTELLI, Andréa; TARANTOLA, Stefano; HOFFMAN, Anders; GIOVANNINI, Enrico. Handbook on constructing 
composite indicators: methodology and user guide. Paris: EC/JRC \& OECD, 2005. Disponível em: $<$ http://www.olis.oecd.org/olis/2005doc.nsf/43bb6130e5e $86 \mathrm{e} 5 \mathrm{fc} 12569 \mathrm{fa} 005 \mathrm{~d} 004 \mathrm{c} / 7 \mathrm{bef}$ 27ea932895d4c1257058004bcdeb/\$FILE/JT00188147.PDF>, Acesso em: 16/02/2007. NISTEP. Science and technology indicators 1994: a systematic analysis of science and technology activities in Japan. NISTEP Report, n.37, 2005. Disponível em: $<\mathrm{http}: / /$ www.nistep.go.jp/achiev/ftx/eng/rep037e/pdf/rep037e.pdf $>$, Acesso em: $13 / 02 / 2007$.

OECD. The measurement of scientific and technical activities: proposed standard practice for surveys of research and experimental development (Frascati Manual). 6ed. Paris: OECD Publications, 2002.

. Proposed guidelines for collecting and interpreting technological innovation data (Oslo Manual). 1 ed. Paris: OECD Publications, 1996. Disponível em: $<$ http://www.oecd.org/dataoecd/35/61/2367580.pdf>, Acesso em: 08 /02/ 2007.

. The measurement of scientific and technical activities: manual on the measurement of human resources devoted to S\&T (Canberra Manual). Paris: OECD Publications, 1995. Disponível em: $<$ http://www.oecd.org/dataoecd/34/0/2096025.pdf>, Acesso em: 08/02/2007.

. The measurement of scientific and technical activities: using patent data as science and technology indicators (Patent Manual). Paris: OECD Publications, 1994. Disponível em: <http://www.oecd.org/dataoecd/33/62/2095942.pdf>, Acesso em: 08/02/2007.

. The measurement of scientific and technical activities: proposed standard method of compiling and interpreting technology balance of payments data (TBP Manual). Paris: OECD Publications, 1990. Disponível em: $<$ http://www.oecd.org/dataoecd/35/13/2347115.pdf>, Acesso em: 08/02/2007.

The measurement of scientific and technical activities: proposed standard practice for surveys of research and experimental development (Frascati Manual). Paris: OECD Publications, 1963.

SCHUMPETER, Joseph (1949). Economic theory and entrepreneurial history. In: Harvard University Research Center in Entrepreneurial History. Change and the Entrepreneur. Cambridge, MA, USA, p.63-84. Reprinted in Revista Brasileira de Inovação, Rio de Janeiro, Brazil, v.1, n.2, p. 203-224, Jul.-Dec. 2002. Disponível em: $<$ http://www.finep.gov.br/revista_brasileira_inovacao/segunda_edicao/ideias_fundadora s_shumpeter.pdf>, Acesso em: $2 \overline{3} / 02 / 2007$.

SMITH, Keith. Science, technology and innovation indicators: a guide for policymakers. Oslo, Norway: IDEA Paper, n.5, STEP Group, 1998. Disponível em: $<$ http://www.step.no/old/Projectarea/IDEA/Idea5.pdf>, Acesso em: 23/02/2007.

SMITH, Keith. Innovation indicators and the knowledge economy: concepts, results and policy challenges. Oslo, Norway: STEP Group, 2000. Disponível em: $<$ http://www.istat.it/istat/eventi/quintaconf/smith.pdf>, Acesso em: 23/02/2007.

STATISTICS CANADA. Blue Sky II - STI indicators at the OECD: lessons learned. Innovation Analysis Bulletin, v.8, n.3, 2006. Disponível em: <http://dsppsd.communication.gc.ca/Collection/Statcan/88-003-XIE/88-003-XIE2006003.pdf>, Acesso em: 02/03/2007. 
UNDP. Human development report 2001 - making new technologies work for human development. Oxford, UK: Oxford University Press, 2001. Disponível em: $<$ http://hdr.undp.org/reports/global/2001/en/pdf/completenew.pdf $>$, Acesso em: 16/02/2007.

UNESCO. Manual for statistics on scientific and technological activities. Paris: UNESCO Office of Statistics. Division of Statistics on Science and Technology, 1984. ST-84/WS/12. Disponível em: $<$ http://www.uis.unesco.org/template/pdf/s\&t/STSManualMain.pdf $>, \quad$ Acesso em: 27/02/2007.

\begin{abstract}
This paper discusses the evolution of the design and production of science, technology and innovation indicators. It is highlighted the most important contributions, weaknesses and perspectives for future improvement of R\&D and innovation indicators, as well as of new experiences (composite, network and positioning indicators). Under the background of the prevailing dynamics and governance structure of the research and innovation systems, theoretical framework and technology context, it is observed the evolution from input and output indicators at the country level to customized indicators, capable of reflecting specificities, needs and policy aims of each actor or category of actors playing in each system.
\end{abstract}

KEYWORDS: Research and Development. Science and Technology. Innovation. Research and innovation networks. Indicators

Originais recebidos em: 08/02/2008

Texto aprovado em: 13/03/2008 